\title{
Resonance at the Rabi frequency in a superconducting flux qubit
}

\author{
Ya. S. Greenberg,, , E. Il'ichev, ${ }^{2}$ G. Oelsner, ${ }^{2}$ and S. N. Shevchenko ${ }^{3,4}$ \\ ${ }^{I}$ Novosibirsk State Technical University, Novosibirsk, Russia \\ ${ }^{2}$ Institute of Photonic Technology, Jena, Germany \\ ${ }^{3}$ B. Verkin Institute for Low Temperature Physics and Engineering, Kharkov, Ukraine \\ ${ }^{4}$ V. Karazin Kharkov National University, Kharkov, Ukraine
}

(Dated: May 26, 2022)

\begin{abstract}
We analyze a system composed of a superconducting flux qubit coupled to a transmission-line resonator driven by two signals with frequencies close to the resonator's harmonics. The first strong signal is used for exciting the system to a high energetic state while a second weak signal is applied for probing effective eigenstates of the system. In the framework of doubly dressed states we showed the possibility of amplification and attenuation of the probe signal by direct transitions at the Rabi frequency. We present a brief review of theoretical and experimental works where a direct resonance at Rabi frequency have been investigated in superconducting flux qubits. The interaction of the qubit with photons of two harmonics has prospects to be used as a quantum amplifier (microwave laser) or an attenuator.

PACS numbers: 85.25.Dq, 85.25.Cp, 85.25.Hv, 84.40.Az
\end{abstract}

\section{INTRODUCTION}

Superconducting qubits are known as the key elements for the solid state implementation of quantum computers ${ }^{1,2}$. Among them superconducting flux qubits are most promising ones due to their relatively long dephasing times and robustness against external disturbances. Since their invention ${ }^{3,4}$ flux qubits have been intensively investigated mainly due to their multiple potential applications, ranging from microwave quantum metamaterials 5 to ultrahighsensitive magnetometers $\underline{6}$.

A superconducting flux qubit (or, simply, flux qubit) is a superconducting loop interrupted by three Josephson junctions. Two junctions are identical while the critical current of the third junction is smaller by approximately twenty percent. In energy language such a structure exhibits two energy levels which are formed by a superposition of quantum states corresponding to clockwise and counter clockwise directions of a superconducting current $I_{q}$ along the loop. The interlevel distance is as follows:

$$
\Delta E=\hbar \sqrt{\varepsilon^{2}+\Delta^{2}},
$$

where $\varepsilon$ is an external parameter which by virtue of external magnetic flux, $\Phi_{X}$ controls the gap between ground and excited states $^{7}$ :

$$
\varepsilon=\frac{2 I_{q}}{\hbar}\left(\Phi_{X}-\frac{\Phi_{0}}{2}\right),
$$

where $\Phi_{0}=h / 2 e$ is a flux quantum. The quantity $\Delta$ in (1) is the gap between ground and excited states at the degeneracy point $(\varepsilon=0)$.

Characteristic energies (in a frequency scale) of superconducting qubits belong to the $\mathrm{GHz}$ frequency range. Nevertheless quantum properties of superconducting flux qubits have been successfully investigated in a $\mathrm{MHz}$ frequency range by so called impedance measuring technique where the flux qubit has been readout with a low frequency (about hundred $\mathrm{MHz}$ ) LC tank circuit ${ }^{8-12}$.
From a formal point of view a superconducting qubit is analogous to a spin-1/2 particle with Hamiltonian

$$
H_{q}=\frac{\hbar \omega_{q}}{2} \sigma_{Z}
$$

where $\omega_{q}=\Delta E / \hbar$.

Many experiments have shown that the interaction between a flux qubit and microwave radiation can be described and interpreted within the models and approaches well known from quantum optics. That is why flux qubits are frequently called artificial atoms. However, there are two significant differences. The first one is that a flux qubit is a macroscopic device, hence, its interaction with microwaves is much stronger than that for the atom-photon interaction. For flux qubit the regime of strong coupling to a photon field can easily be achieved. For this reason the effects which are observed in a media with many atoms can be observed for a single flux qubit interacting with a microwave field ${ }^{13}$. The second difference relates to the form of the interaction between a flux qubit and a photon field. The interaction Hamiltonian is as follows 24 .

$$
H_{i n t}=\hbar g\left(\frac{\varepsilon}{\omega_{q}} \sigma_{Z}+\frac{\Delta}{\omega_{q}} \sigma_{X}\right)\left(a^{+}+a\right),
$$

where $a^{+}, a$ are creation and annihilation operators for photons, $g$ is the coupling strength between qubit and photon field.

Hamiltonian (4) contains the "longitudinal" term $\hbar g \varepsilon \sigma_{Z}\left(a^{+}+a\right) / \omega_{q}$ the analogue of which is absent in quantum optics due to antisymmetric properties of the atom dipole moment. This term gives rise to the transitions between Rabi levels of the same doublet, the effect which is not allowed for atoms in photon field. This effect was first observed long time ago in low frequency NMR experiments ${ }^{14,15}$ where a time dependent part of the polarizing magnetic field $B_{0}$ played the role of our parameter $\varepsilon$ in (4) (see also section VIIB in ${ }^{16}$ ).

The first experimental detection of the voltage fluctuations at the Rabi frequency in low frequency LC circuit coupled to 
a flux qubit was observed in ${ }^{18}$. This paper gave rise to a series of theoretical works devoted to the transitions between Rabi levels in superconducting flux qubit. $\operatorname{In}^{19}$ it was shown that in the dissipative two level system it is possible to obtain persistent Rabi oscillations excited by an external signal which is in resonance with Rabi splitting. It was also shown that in a dissipative two level system the regimes of amplification and attenuation of a probe signal due to transitions between Rabi levels of the same pair were possible ${ }^{16,17,20,21}$. In these papers two- probe spectroscopy was considered with a driving signal controlling the populations of the Rabi levels. In Ref ${ }^{16}$ the probe signal was considered as classical wave, while in the works ${ }^{20,21}$ the probe signal was a photon field. All these theoretical findings have been confirmed in experiment ${ }^{22}$ where a flux qubit driven by a high-frequency field with frequency $\omega_{d}$ was coupled through a mutual inductance to a low-frequency tank circuit with frequency $\omega_{T}$ much lower than the qubit gap $\Delta$. In this experiment the amplification and attenuation of the LC voltage by a flux qubit are clearly due to the transitions between nearby Rabi levels with the splitting being in resonance with the LC tank.

With the progress in thin film technology it became possible to develop a microstrip waveguide with a very high quality factor $^{23}$. This, in turn, paved the way to many interesting experiments where one or several qubits were coupled on-chip to a microwave resonator (see, for example, Ref. ${ }^{24}$ and references therein). In the context of the subject of this review it is necessary to mention the paper ${ }^{25}$ where the first experimental demonstration were given of the amplification and attenuation of microwaves due to Rabi transitions between the levels of the same Rabi doublet. In Ref. ${ }^{25}$ a flux qubit was placed in the middle of coplanar half-wavelength waveguide which had two gaps at its ends. For this reason the waveguide plays for microwaves the same role as a Fabry-Perot resonator plays for photons in quantum optics. The waveguide was driven with a strong signal at its third harmonic. A weak probe signal at the fundamental frequency was detected at the output where the phase and the amplitude of the transmission factor were measured. Because the Rabi splitting can be controlled by several external parameters it can be matched to resonance with the fundamental frequency resulting in attenuation or amplification of the probe signal. The direction of this effect depends on the populations of the Rabi levels which in turn depend on the external parameters and on the rates of relaxation and dephasing of the flux qubit. This experiment has been carefully analyzed in $\operatorname{Ref}^{26}$. Here we give a brief account of this analysis from the point of the dressed state approach which is well known in quantum optics (see, for example, Ref. ${ }^{29}$ ).

\section{SUPERCONDUCTING FLUX QUBIT AND PHOTON FIELD}

\section{A. Interaction of a superconducting flux qubit with a strong driving microwave field}

First we consider a superconducting flux qubit which interacts with a one-mode quantized driving photon field.

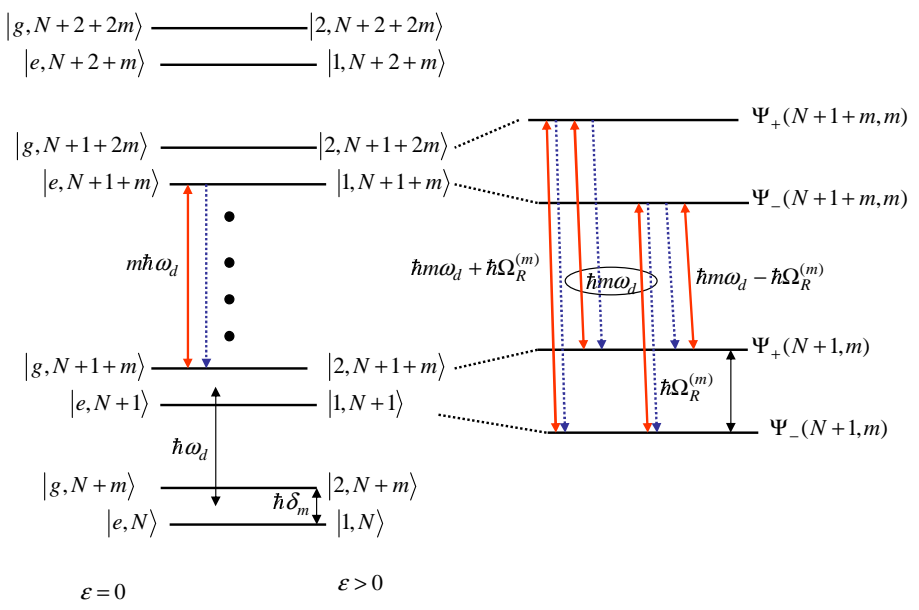

FIG. 1: (a) A ladder of the energy doublets which represents the uncoupled qubit- photon system $(\varepsilon=0)$ and the system where photons are coupled to the longitudinal term $(\varepsilon>0)$. Here $\delta_{m}=$ $\omega_{q}-m \omega_{d}<0$. Spontaneous and stimulated transitions at $m \omega_{d}$ are shown by dotted (blue) and red arrows, respectively. (b) Two doublets of the singly dressed states of the infinite ladder of energy levels. Sideband transitions at $\omega_{d} \pm \Omega_{R}^{(m)}$ and central transition at $\omega_{d}$ are shown by red solid arrows. Corresponding spontaneous transitions are shown by dotted arrows.

$$
\begin{gathered}
H_{q b-d}=\frac{\hbar \omega_{q}}{2} \sigma_{Z}+\hbar \omega_{d} d^{+} d+\hbar g_{d} \frac{\varepsilon}{\omega_{q}} \sigma_{Z}\left(d^{+}+d\right)+ \\
+\hbar g_{d} \frac{\Delta}{\omega_{q}} \sigma_{X}\left(d^{+}+d\right)
\end{gathered}
$$

where $d^{+}, d$ are creation and annihilation operators for the field mode with an angular frequency $\omega_{d}$. The third and fourth terms represent the coupling between the flux qubit and the driving field with $g_{d}$ being the coupling strength.

We define the uncoupled basis for Hamiltonian (5) as a direct product of the qubit ground $|g\rangle$ and excited $|e\rangle$ states and Fock states $|N\rangle$ for $N$ driving photons: $|e, N\rangle,|g, N\rangle$. The energy levels for the uncoupled qubit-photon system are $E_{ \pm N}=\hbar \omega_{d} N \pm \hbar \omega_{q} / 2$. Having in mind to consider the multiphoton transitions we assume the frequency of the driving field to be close to a fraction of the qubit frequency $\omega_{d} \approx$ $\omega_{q} / m$, where $m$ is a positive integer $(m=1,2 \text {, etc. })^{27,28}$. The structure of the energy levels for uncoupled qubit- photon system for $\delta_{m}=\omega_{q}-m \omega_{d}<0$ is shown at the left side of Fig 1a. This structure is a ladder of doublets split by $\hbar \delta_{m}$ and separated by $\hbar \omega_{d}$.

The first three terms of Hamiltonian (5) can be reduced to diagonal form exactly ${ }^{29}$. The eigenenergies and eigenfunctions are as follows :

$$
E_{1 N}=\frac{\hbar \omega_{q}}{2}+\hbar \omega_{d} N-\frac{\hbar g_{d}^{2} \varepsilon^{2}}{\omega_{q}^{2} \omega_{d}}
$$




$$
\begin{gathered}
E_{2 N}=-\frac{\hbar \omega_{q}}{2}+\hbar \omega_{d} N-\frac{\hbar g_{d}^{2} \varepsilon^{2}}{\omega_{q}^{2} \omega_{d}} \\
|1 N\rangle=e^{-\lambda\left(d^{+}-d\right)}|e N\rangle \\
|2 N\rangle=e^{\lambda\left(d^{+}-d\right)}|g N\rangle
\end{gathered}
$$

where $\lambda=g_{d} \varepsilon / \omega_{q} \omega_{d}$.

Remarkably, the account for the third term in Eq. (5) results in essentially the same energy levels as those for the uncoupled system if we disregard the constant downshift term in Eqs. (6) and (7). We show these energy levels at the right side of Fig 11 with the new eigenfunctions $|1, N\rangle,|2, N\rangle$ attributed to them.

The efficient coupling of the driving field with the qubit is caused by the fourth term in Hamiltonian (5). It lifts the degeneracy of the levels $E_{1 N_{1}}$ and $E_{2 N_{2}}$ with $\omega_{q} \approx m \omega_{d}$, where $m=N_{2}-N_{1}$ and gives rise to the well known dressed energy spectrum shown in Fig 1b (see, for example ${ }^{29}$ and references therein).

The calculation of the corresponding matrix element yields the following result:

$$
\begin{gathered}
\left\langle 1, N\left|\sigma_{X}\left(d^{+}+d\right)\right| 2, N+m\right\rangle= \\
=\exp \left(-2 \lambda^{2}\right)\left(\frac{m}{\lambda}-4 \lambda\right) J_{m}(4 \lambda \sqrt{\langle N\rangle})
\end{gathered}
$$

In Eq. (10) $J_{m}$ is the Bessel function, $\langle N\rangle$ is the average number of driving photons which is assumed to be large: $N_{1}, N_{2} \approx\langle N\rangle>>m$.

Hence, for the energy splitting between degenerate states we obtain:

$$
E_{ \pm}(N, m)=\hbar \omega_{d} N \pm \frac{1}{2} \hbar \Omega_{R}^{(m)}
$$

where,

$$
\Omega_{R}^{(m)}=\sqrt{\left(\omega_{q}-|m| \omega_{d}\right)^{2}+4 \Lambda_{m}^{2}}
$$

is the $m$ - photon Rabi frequency with

$$
\Lambda_{m}=g_{d} \frac{\Delta}{2 \omega_{q}} \frac{m}{\lambda} J_{m}(4 \lambda \sqrt{\langle N\rangle})
$$

which follows from (10) for $\lambda<<1$.

This Bessel-like behavior of the Rabi splitting can be obtained also classically and, in fact, is known for a long time (see, for example, Ref ${ }^{30}$ ). It has also been used for the study of quantum dynamics in the microwave irradiated superconducting charge qubits ${ }^{31}-34$. However, due to the presence of the photon operator $\left(d^{+}+d\right)$, the matrix element (10) differs from that for a charge qubit, and from that considered in Ref. $\frac{29}{}$.

Similar to the uncoupled spectrum the dressed spectrum consists of a series of doublets split by $\hbar \Omega_{R}^{(m)}$ and separated by $\hbar \omega_{d}$. As is evident from Eq. (11), the splitting between dressed levels can be effectively adjusted by three external parameters: the external magnetic flux, $\Phi_{X}$ which is hidden in $\varepsilon$, the frequency of the driving field, $\omega_{d}$, and the input power, which is proportional to $\langle N\rangle$. For reasons which will be clear later we call these dressed states as singly dressed states (SDS).

At the degeneracy point $(\epsilon=0)$ only single photon transition $(m=1)$ survives

$$
\Omega_{R}^{(1)}=\sqrt{\left(\omega_{q}-\omega_{d}\right)^{2}+\frac{4 g_{d}^{2} \Delta^{2}\langle N\rangle}{\omega_{q}^{2}}}
$$

The state vectors for two states of a doublet are superposition states of the unperturbed states $\left|1, N_{1}\right\rangle$ and $\left|2, N_{2}\right\rangle$ where $N_{2}-N_{1}=m$ :

$$
\Psi_{ \pm}(N, m)=\alpha_{ \pm}^{(m)}|1, N\rangle+\beta_{ \pm}^{(m)}|2, N+m\rangle
$$

where

$$
\alpha_{ \pm}^{(m)}=\frac{-\sqrt{2} \Lambda_{m}}{\sqrt{\Omega_{R}^{(m)}\left(\Omega_{R}^{(m)} \mp \delta_{m}\right)}}
$$

$$
\beta_{ \pm}^{(m)}=\frac{\delta_{m} \mp \Omega_{R}^{(m)}}{\sqrt{2 \Omega_{R}^{(m)}\left(\Omega_{R}^{(m)} \mp \delta_{m}\right)}}
$$

and $\delta_{m}=\omega_{q}-|m| \omega_{d}$ is the detuning between the qubit frequency and the $m$ photon driving signal frequency.

There are three types of transitions between levels shown in Fig 1] the spontaneous emission, the absorption, and the stimulated emission caused by an external probe signal. The allowed transitions occur only between the states with nonzero matrix element of $\sigma_{X}$ which is a part of the qubit "dipole" operator $\varepsilon \sigma_{Z} / \omega_{q}+\Delta \sigma_{X} / \omega_{q}$ which describes the qubit interaction with the photon field (4).

Spontaneous emission is due to the transition between qubit states with the same number of driving photons $|e, N\rangle \rightarrow$ $|g, N(|1, N\rangle \rightarrow \mid 2, N)$. In Fig[1 this transition is shown by the blue dotted arrow. In the picture of dressed states this transition corresponds to two sidebands at the frequencies $m \hbar \omega_{d} \pm \hbar \Omega_{R}^{(m)}$ and one central line $m \hbar \omega_{d}$ which are shown in Fig 1b by the blue dotted arrows. This is well known fluorescent Mollow triplet ${ }^{35}$. Absorption and stimulated emission are shown in Fig 1 by the red solid arrows. These transitions occur between the same levels which are connected by the spontaneous transitions. Which process is dominant at the given transition, the absorption or the stimulated emission, or, in other words, whether the probe signal is amplified or attenuated depends on the population of the corresponding levels.

By using superconducting qubits as artificial atoms these effects at sideband Rabi transitions between SDS have been demonstrated experimentally. A lasing action with a single Josephson junction charge qubit has been realized ${ }^{36}$ and the stimulated emission by a flux qubit was successfully employed for the amplification of a microwave signal passing through an open transmission line ${ }^{37}$. The Rabi sidebands 
have been observed for different structures of superconducting qubits coupled to a microwave transmission line: one photon sidebands for three level dressed states in a transmon qubit ${ }^{38}$, two-photon sidebands for a Cooper pair box ${ }^{39}$ and multiphoton sidebands for a flux qubit ${ }^{40}$.

Therefore, in principle, superconducting qubits allow one to obtain the amplification (attenuation) of a probe signal at the Rabi sideband transitions, which are similar to those in quantum optics.

\section{B. Interaction of the driven flux qubit with a quantized photon field in a coplanar waveguide resonator}

In this section we consider the effect of amplification (attenuation) of a probe microwave signal which is due not to the sideband Rabi transitions, but to the transitions directly at the Rabi frequency. In spite of some analogy with quantum optics, there is an essential difference: in quantum optics direct transitions at the Rabi frequency are forbidden at least in the first order perturbation since the matrix element of the dipole interaction between Rabi levels is exactly equal to zero. As opposed to optical systems, solid state quantum systems are scalable and tunable, therefore the effect of direct Rabi transitions can be used for microwave quantum engineering 41 .

We place the system qubit+ driving field (5) in a photon resonator (low loss rate coplanar waveguide) with the frequency $\omega_{0}$ being close to Rabi frequency $\Omega_{R}^{(m)}$. This results in the total Hamiltonian, $H=H_{q b-d}+H_{r}$, where

$H_{r}=\hbar \omega_{0} a^{+} a+\hbar g_{r} \frac{\varepsilon}{\omega_{q}} \sigma_{Z}\left(a^{+}+a\right)+\hbar g_{r} \frac{\Delta}{\omega_{q}} \sigma_{X}\left(a^{+}+a\right)$

Here $g_{r}$ is the qubit resonator coupling; $a^{+}, a$ are creation and annihilation operators for photons in the resonator, and we assume that the number $n$ of resonator photons is small $(n<<$ $N)$.

Since the frequency of the resonator $\omega_{0}$ is assumed to be smaller than the qubit gap $\Delta$, the resonator cannot excite the qubit. Therefore we neglect the $\sigma_{X}$ term in Eq. (17). The second term in Eq. (17) evidently, leads to the transition between two levels within a dressed doublet (Fig 1b), a transition which is not allowed for $\sigma_{X}$. It means that a probe signal which is matched to the splitting between the dressed states of the same doublet can cause resonant transitions between them. This leads to the emission or absorption of Rabi photons depending on the populations of these nearby levels resulting in the energy exchange between the flux qubit and the resonator. Below we analyze this resonant effect within the frame of doubly dressed states (DDS).

We define, analogous to Eqs. (8) and (9), the wave functions $|1 N n\rangle=e^{-\lambda\left(d^{+}-d\right)}|e N n\rangle$ and $|2 N n\rangle=$ $e^{\lambda\left(d^{+}-d\right)}|g N n\rangle$ with the eigenenergies $E_{1 N}=\hbar \omega_{q} / 2+$ $\hbar \omega_{d} N+\hbar \omega_{0} n, E_{2 N}=-\hbar \omega_{q} / 2+\hbar \omega_{d} N+\hbar \omega_{0} n$, where $n$ is the number of Rabi photons in the resonator. Furthermore, the wave functions $\Psi_{ \pm}(N, m) \otimes|n\rangle$ with $n$ photons we denote as $\Psi_{ \pm}(N, m, n)$. If the resonator frequency $\omega_{0}$ is close to the Rabi frequency $\Omega_{R}^{(m)}$ we get an energy spectrum a part of which is shown in Fig 2 . These doublets fill the gap between two neighbor doublets of the dressed states.

The additional degeneracy for the levels $\Psi_{-}(N, m, n+1)$ and $\Psi_{+}(N, m, n)$ is lifted by the second term in the resonator Hamiltonian (17). For the energy splitting between these new degenerate states we find:

$$
E_{ \pm}(N, m, n)=\hbar \omega_{d} N+\hbar \omega_{0} n \pm \frac{1}{2} \hbar \Omega_{R}^{(n)}
$$

where

$$
\Omega_{R}^{(n)}=\sqrt{\left(\Omega_{R}^{(m)}-\omega_{0}\right)^{2}+4 F_{n}^{2}}
$$

with

$$
F_{n}=\frac{2 \Lambda_{m}}{\Omega_{R}^{(m)}} \frac{g_{r} \varepsilon}{\omega_{q}} \sqrt{n+1}
$$

The resulting wave functions are

$$
\Phi_{ \pm}(N, m, n)=A_{ \pm} \Psi_{+}(N, m, n)+B_{ \pm} \Psi_{-}(N, m, n+1)
$$

where

$$
\Psi_{ \pm}(N, m, n)=\alpha_{ \pm}^{(m)}|1, N, n\rangle+\beta_{ \pm}^{(m)}|2, N+m, n\rangle
$$

with $\alpha_{ \pm}^{(m)}, \beta_{ \pm}^{(m)}$ being given in Eqs. 15, 16). The quantities $A_{ \pm}, B_{ \pm}$in Eq. (20) are as follows:

$$
\begin{gathered}
A_{ \pm}=\frac{-\sqrt{2} F}{\sqrt{\Omega_{R}^{(n)}\left(\Omega_{R}^{(n)} \mp \delta_{R}\right)}} \\
B_{ \pm}=\frac{\delta_{R} \mp \Omega_{R}^{(n)}}{\sqrt{2 \Omega_{R}^{(n)}\left(\Omega_{R}^{(n)} \mp \delta_{R}\right)}}
\end{gathered}
$$

where $\delta_{R}=\Omega_{R}^{(m)}-\omega_{0}$.

Two pairs of states 20 are shown in Fig $2 \mathrm{~b}$. These states are called the doubly dressed states since they are obtained by the dressing of the previously dressed states (Fig 1b) with the interaction between the qubit and the photons in in the resonator.

The longitudinal term in the qubit dipole operator, $\sigma_{Z}$, of the probe signal induces the transitions between singly dressed states $\Psi_{+}(N, m, n)$ and $\Psi_{-}(N, m, n)$. An example of such a transition is shown by the red arrow in Fig 2 a. In the picture of doubly dressed states (Fig $2 b$ ) this transition corresponds to two sideband lines with the frequencies $\Omega_{R}^{(m)} \pm \Omega_{R}^{(n)}$, and a central line $\Omega_{R}^{(m)}$. If $\omega_{0}=\Omega_{R}^{(m)}$ we get the only line at $\Omega_{R}^{(n)}$. Hence, the transition between the states of singly dressed doublet transforms to sideband transitions between neighbor doublets of doubly dressed states. 
(a)

(b)

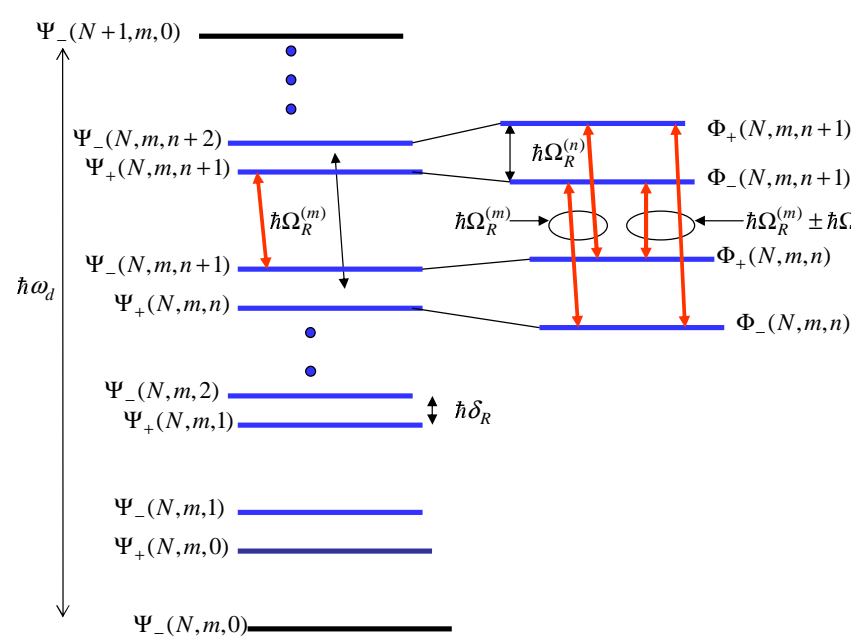

FIG. 2: (a) Doublets of the uncoupled system of singly dressed states and $n$ - photon resonator $\left(\delta_{R}=\Omega_{R}^{(m)}-\omega_{0}<0\right)$. The allowed transition induced by the longitudinal term $\sigma_{Z}$ is shown by the red arrow. (b) Two pairs of the doubly dressed states. Two sidebands at $\Omega_{R}^{(m)} \pm \Omega_{R}^{(n)}$ and a central line at $\Omega_{R}^{(m)}$ are shown by red arrows.

\section{TRANSMISSION FACTOR. AMPLIFICATION AND ATTENUATION OF A PROBE SIGNAL}

There are several external parameters which can be used to match the splitting $\Omega_{R}^{(m)}$ between Rabi levels with the fundamental frequency $\omega_{0}$. These are the external magnetic flux $\Phi_{X}$ (2), the driving frequency $\omega_{d}$, and the input driving amplitude $A_{d}=g_{d} \sqrt{\langle N\rangle}$.

While it is not difficult to find the positions of the resonance from the equation $\omega_{0}=\Omega_{R}^{(m)}$, it is not easy to calculate the transmission factor which shows the amplification or attenuation of the probe signal. The reason for this is that the dynamics of the qubit is essentially dissipative. The bare qubit's relaxation, $\Gamma$ and decoherence, $\Gamma_{\varphi}$ rates are transformed in a complicated way under influence of the interaction of the qubit with photon fields ${ }^{21,26}$. These effects of dissipation can be accounted for by the Liouville equation for the density matrix of the system, including the relevant damping terms, which are assumed to be of Markovian form²6.

The transmission factor, which is the output-to-input ratio for a probe signal, is defined in the way adopted in quantum optics 26 :

$$
t=i \frac{\hbar \kappa}{2 \xi_{p}}\langle a\rangle
$$

where $\kappa$ is the loss rate of photons in the coplanar waveguide, $\xi_{p}$ the amplitude of the probe signal, $\langle a\rangle=\operatorname{Tr}(a \rho), \rho$ the density matrix of the system, and the trace over the photon states of the fundamental mode $|n\rangle$ and the two qubit states $| \pm\rangle$.
Here we show two plots which have been calculated with the account of dissipation effects ${ }^{26}$. In Fig 3 (left) we show the transmission amplitude $|t|$ of the fundamental-mode signal as a function of the qubit's bias $\varepsilon$ and the detuning $\omega_{p}-\omega_{0}$ from the probing frequency $\omega_{p}$. When the frequency of the probing signal is close to the Rabi frequency $\Omega_{R}^{(m)}$ a resonant energy exchange between the qubit and the fundamental mode ${ }_{R}^{(n)}$ of the resonator results in amplification or attenuation of the transmitted signal. The observation of such amplification was recently reported in 25 .

The effect of amplification and attenuation of the transmitted signal can also be related to the increase or decrease of the intracavity photon number. This is demonstrated in Fig 3 (right), where the stationary solution for the intracavity photon number is plotted as the function of the qubit's bias $\varepsilon$ and the driving amplitude $A_{d}$.

These calculations were done in the regime of the weak driving for the following parameters $\frac{25}{}$ : $\Delta=3.7 \mathrm{GHz}, g_{r}=$ $0.8 \mathrm{MHz}, \omega_{0} / 2 \pi=2.5 \mathrm{GHz}, \Gamma=80 \mathrm{MHz}, \Gamma_{\varphi}=10 \mathrm{MHz}, A_{d}$ $=7 \mathrm{GHz}, \omega_{d}=3 \omega_{0}, \kappa=30 \mathrm{kHz}$, and $\xi_{p}=0.5 \kappa$.

Below we show the results of a recent experiment where a substantial amplification of a probe signal is demonstrated. We used a sample similar to the one in ${ }^{42}$. The resonator is probed at its fundamental mode with a frequency of $\omega_{0} / 2 \pi \approx$ $2.5 \mathrm{GHz}$ and driven at its 5 th harmonic with $\omega_{d}=5 \omega_{0}$. If the transmission is monitored with low driving powers, only the dispersive shift of the resonator frequency close to the qubits degeneracy point is observed. No resonant interaction occur since the energy gap of the qubit $\Delta \approx 6 \mathrm{GHz}$ is bigger than the eigenfrequency of the resonators fundamental mode. This can be seen in Fig. 4 at the upper, light curve, which is measured for low driving powers.

When the driving power is increased (to the darker and lower curves in Fig. (4), signatures of resonant interactions appear. They correspond to Rabi resonances between the dressed states and the resonator fundamental mode and manifest themselves as dips and peaks in Fig. 4(a) and as char-

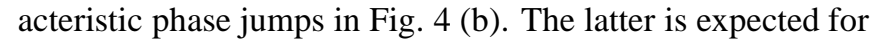
avoided crossings of the energy levels of the resonator (compare for example ${ }^{42}$ ). The amplification peaks occur, where the qubit energy is smaller than the driving frequency, where $|\epsilon|<$ $11 \mathrm{GHz}$, and vice versa for the attenuation. This is because the population depends on the sign of the detuning between the two, as explained in detail in 25,26 . The shift of the resonance points follows the resonance condition $\Omega_{R}^{(1)}=\omega_{0}$. Note, if the amplitude term in (13) dominates, the detuning is close to zero to fulfill the resonance condition. Then the populations get equalized and a wide dip instead of a peak-dip-structure is expected due to the frequency shift in resonance. Finally we mention that not only the position of the peaks, but also their height depends on the driving power. To find optimal parameters for the amplification we analyzed the power dependencies for one of the amplification points as presented in Fig. 5.

As seen in Fig. 5. (a) the probing power has, in the analyzed region, almost no influence on the position of the resonance point. Only for high powers above $-140 \mathrm{dBm}$ a small shift to bigger values of $|\epsilon|$ is observed. Nevertheless, as also the amplification effect vanishes in this region no further investi- 

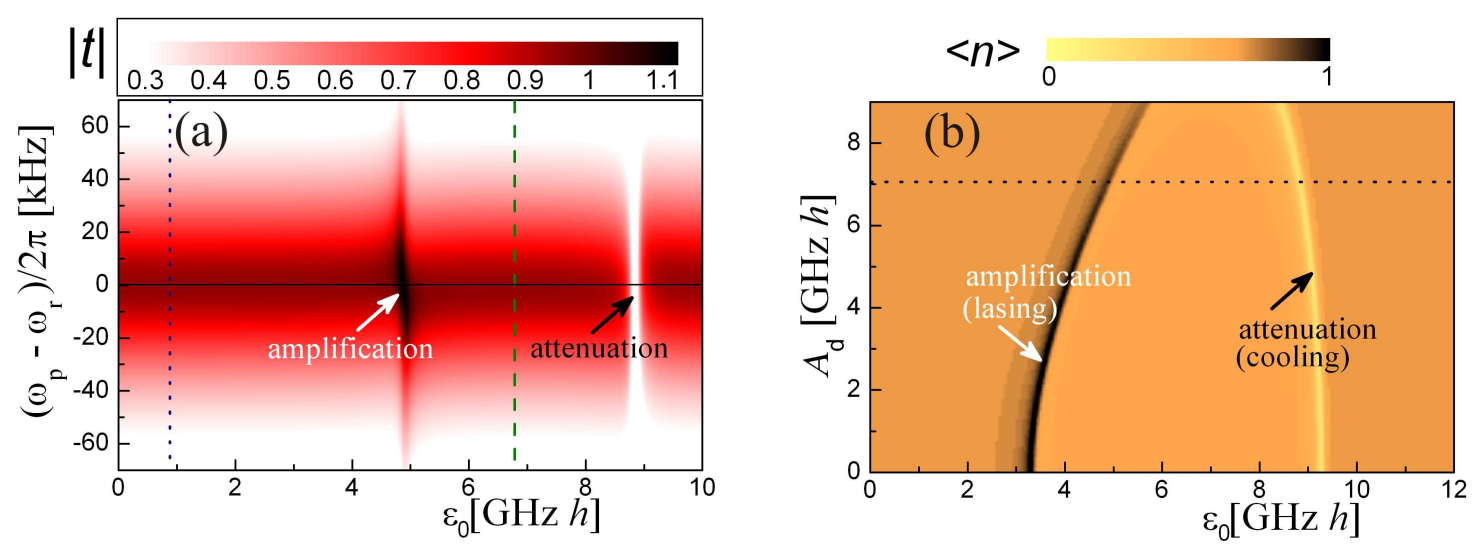

FIG. 3: (left) Normalized transmission amplitude $|t|$ for the fundamental- mode signal versus the bias $\varepsilon$ and the probing frequency detuning $\omega_{p}-\omega_{0}$. The amplification and attenuation of the transmitted signal is displayed as the increase or decrease of the transmission at resonance $\omega_{p} \approx \omega_{0}$; (right) The average intracavity photon number $\langle n\rangle$ versus the bias $\varepsilon$ and driving amplitude $A_{d}$. The value of the driving amplitude, at which left plot of the Fig 3 was calculated, is shown by the dashed line. The dark and light colors correspond to the increase and decrease of the intracavity photon number which, can be termed as lasing or cooling of the resonator and results in the amplification or attenuation of the transmitted probing signal. Here $\omega_{p}=\omega_{0}$.
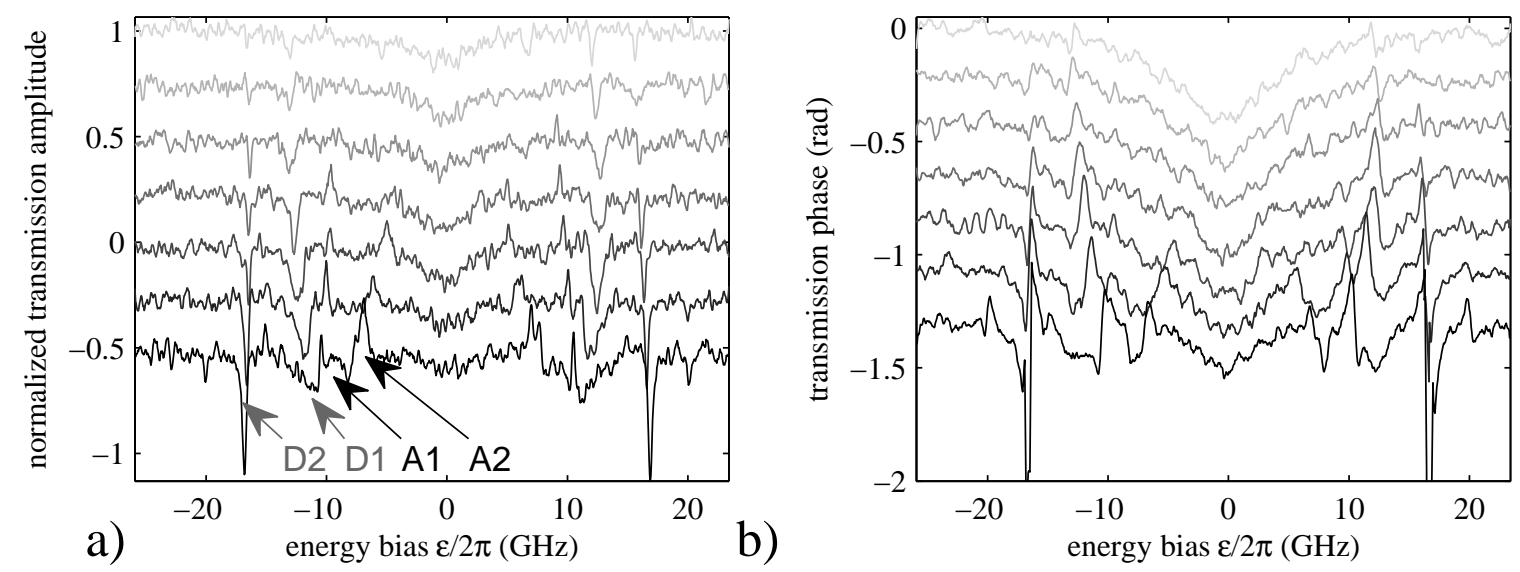

FIG. 4: Normalized transmission amplitude (a) and phase (b) of a weak probe signal at $\omega_{p}=\omega_{0}$ through the resonator. From light to dark color the driving power is increased in $2 \mathrm{dBm}$ steps. The curves are shifted from another by 0.25 for the amplitude and 0.2 rad for the phase for better visibility. In (a) the peaks for the one (A1) and two (A2) photon amplification and similar the dips of the attenuation (D1 and D2) are marked.

gations for higher powers where done. The gain of the feature has a monotonic increase for decreasing probing powers. This may be misleading since the resonant process can add a constant number of photons in each cycle of the resonator. Only the reference signal is reduced and since the transmission is given as the ratio of the output to the input amplitude the described dependence is well expected. The driving power in turn has influence on both the position and the strength of the amplification, as seen in Fig. 5 (b). As already briefly discussed the position follows the resonance condition. Therefore, if the amplitude dependent term of the Rabi splitting is increased the detuning between the driving signal and the qubit needs to be reduced. Also a clear optimum in the amplification process is observed. This has two reason, on the one hand the effective coupling between the Rabi levels and the resonator is increased with increasing driving amplitude (see (19) or ${ }^{25,26}$ ), but also as explained above the population difference between the states is decreased (see also the dissipative dynamics in ${ }^{20.26}$ ). In addition to the increasing coupling also the eigenfrequency of the resonator at the Rabi frequency is shifted more strong. Then by measuring only for $\omega_{0} / 2 \pi=$ const $=2.5 \mathrm{GHz}$ we not always measure in the maximum of the resonators Lorentzian, which can hide the amplification.

\section{CONCLUSION}

We have considered a superconducting flux qubit placed in a microwave cavity resonator. The qubit is irradiated by a strong driving field at a higher harmonic and is probed by 

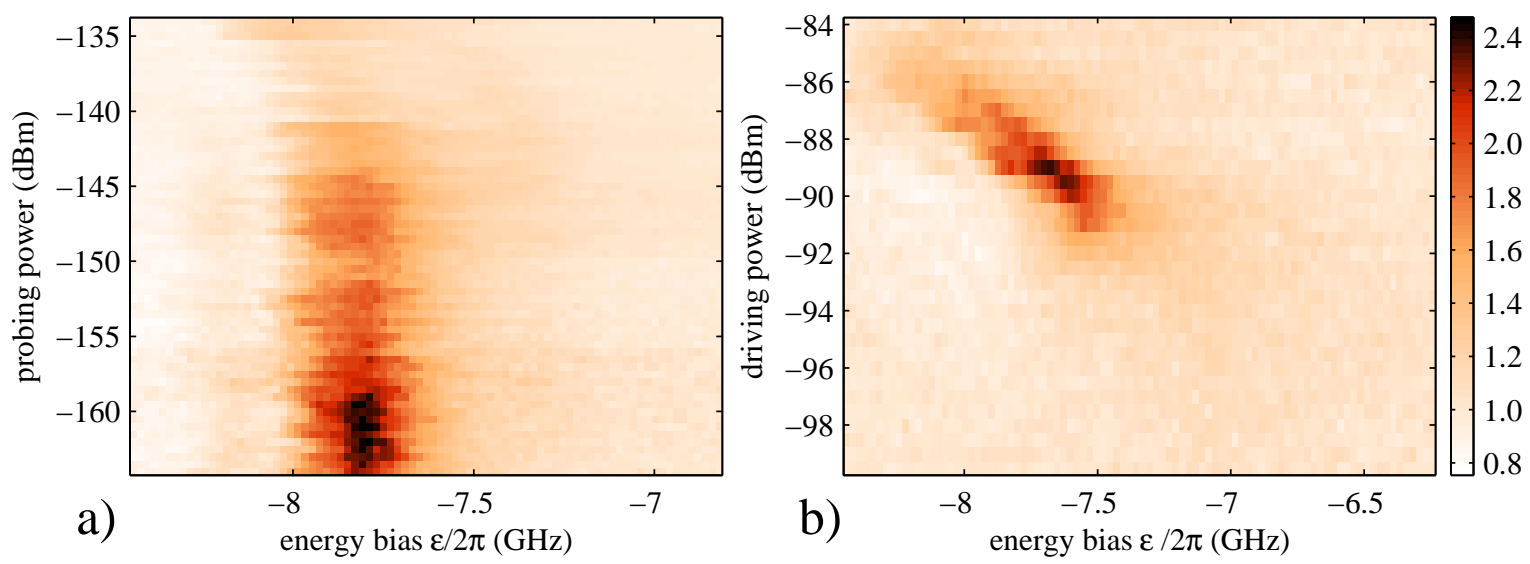

FIG. 5: Dependence of the amplification on the probing (a) and driving (b)power at the input of the resonator measured at $\omega_{0}$. The colormap for both ofthe plots is the same and the corresponding normalized transmission is given in the left colorbar.The left plot is measured with a driving power of $-90 \mathrm{dBm}$ and the right plot with a probingpower of $-160 \mathrm{dBm}$.

a weak signal at its fundamental frequency (first harmonic). The driving signal is not observed directly and is included into the considerations by the qubit's dressed states. Similarly, the interaction of the dressed qubit with the resonator's fundamental mode can be described as the second dressing of the qubit's dressed states. When the energy of the probing photons matches the dressed energy levels, a resonant energy exchange between the qubit and the resonance cavity results in either the amplification or the attenuation of the probing signal, depending on the mutual populations of Rabi levels, which, in turn, depend on the frequency detuning and on the tunable relaxation rates.

We briefly reviewed the major theoretical and experimental activity in this field and presented recent calculations and experimental results which demonstrate experimentally the main features of the dressed-state amplification of a probe field by a strongly driven qubit directly at the Rabi frequency. We believe that further investigation of this effect could find poten- tial applications in the development of new types of quantum microwave amplifiers and sources.

\section{Acknowledgments}

This research has received funding from the European Community's Seventh Framework Programme (FP7/20072013) under grant agreement No. 270843. The work was partly supported by NAS of Ukraine (Project No. 4/14 NANO), DKNII (Project No. M/231-2013), BMBF (UKR2012-028). Ya.S.G. acknowledges the financial support from the Russian Ministry of Education and Science within the framework of the project of the state assignment, entitled "Development of the basic components for quantum microwave circuits".
* Electronic address: yakovgreenberg@yahoo.com

1 J. Clarke and F. K. Wilhelm Superconducting quantum bits. $\mathrm{Na}$ ture 453, 1031 (2008).

2 M. Grajcar, A. Izmalkov, E. Il'ichev Possible implementation of adiabatic quantum algorithm with superconducting flux qubits. Phys. Rev. B71, 144501 (2005).

3 J. E. Mooij, T. P. Orlando, L. Levitov, Lin Tian, C. H. van der Wal, S. Lloyd Josephson Persistent-Current Qubit. Science 285, 1036 (1999).

4 T. P. Orlando, J. E. Mooij, Lin Tian, C. H. van der Wal, L. S. Levitov, S. Lloyd, J. J. Mazo Superconducting persistent-current qubit. Phys. Rev. B60, 15398 (1999).

5 P. Macha, G. Oelsner, J.-M. Reiner, M. Marthaler, S. Andre, G. Schoen, U. Huebner, H.-G. Meyer, E. Il'ichev, and A. V. Ustinov Implementation of a Quantum Metamaterial. arXiv: 1309.5268 [quant-ph](2013).

${ }^{6}$ E. Il'ichev and Ya. S. Greenberg, Flux qubit as a sensor of mag- netic flux. Europhysics Letters 70, 58005 (4 pp.) (2007).

${ }^{7}$ Caspar H. van der Wal, A. C. J. ter Haar, F. K. Wilhelm, R. N. Schouten, C. J. P. M. Harmans, T. P. Orlando, Seth Lloyd, and J. E. Mooij Quantum Superposition of Macroscopic Persistent-Current States. Science 290, 773 (2000).

${ }^{8}$ E. Il'ichev, Th. Wagner, L. Fritzsch, J. Kunert, V. Schultze, T. May, H. E. Hoenig, H.G. Meyer M. Grajcar, D. Born, W. Krech M. V. Fistul and A. M. Zagoskin Characterization of superconducting structures designed for qubit realizations. Appl. Phys. Lett. 80, 4184 (2002).

9 Ya. S. Greenberg, A. Izmalkov, M. Grajcar, E. Il'ichev, W. Krech, H.-G. Meyer, M. H. S. Amin, and A. Maassen van den Brink Lowfrequency characterization of quantum tunneling in flux qubits. Phys. Rev B 66, 214525 (2002).

10 Ya. S. Greenberg, A. Izmalkov, M. Grajcar, E. Il'ichev, W. Krech, and H.-G. Meyer Method for direct observation of coherent quantum oscillations in a superconducting phase qubit. Phys. Rev B66, 
224511 (2002)

11 M. Grajcar, A. Izmalkov, E. Il'ichev, Th. Wagner, N. Oukhanski, U. Hübner, T. May, I. Zhilyaev, H. E. Hoenig, Ya. S. Greenberg, V. I. Shnyrkov, D. Born, W. Krech, H.-G. Meyer, A. Maassen van den Brink, M. H. S. Amin Low-frequency measurement of the tunneling amplitude in a flux qubit. Phys. Rev. B69, 060501(R)3 pp. (2004).

12 E. Il'ichev, A. Yu. Smirnov, M. Grajcar, A. Izmalkov, D. Born, N. Oukhanski, Th. Wagner, W. Krech, H.-G. Meyer, and A. Zagoskin Radio-frequency method for investigation of quantum properties of superconducting structures. Low Temp. Phys. 30, 620 (2004).

${ }^{13}$ O. Astafiev, A. M. Zagoskin, A. A. Abdumalikov Jr., Yu. A. Pashkin, T. Yamamoto, K. Inomata, Y. Nakamura, J. S. Tsai Resonance Fluorescence of a Single Artificial Atom. Science 327, 840 (2010).

14 A. E. Mefed and V. A. Atsarkin, Pis'ma Zh. Eksp. Teor. Fiz. 25, 233 (1977) [JETP Lett. 25, 215-217. (1977)].

15 A. E. Mefed and V. A. Atsarkin Direct observation of NMR in a rotating coordinate system and supression of nuclear dipole interactions in a solid. Zh. Eksp. Teor. Fiz. 74, 720 (1978) [Sov. Phys. JETP 47, 378-385 (1978)].

16 Ya. S. Greenberg Low-frequency Rabi spectroscopy of dissipative two-level systems: Dressed-state approach. Phys. Rev. B76, 104520 (2007)

17 Ya. S. Greenberg and E. Il'ichev Quantum theory of the lowfrequency linear susceptibility of interferometer-type superconducting qubits. Phys. Rev. B77, 094513 (2008).

18 E. Il'ichev N. Oukhanski, A. Izmalkov, Th. Wagner, M. Grajcar, H.-G. Meyer, A.Yu. Smirnov, Alec Maassen van den Brink, M. H. S. Amin, and A. M. Zagoskin Continuous Monitoring of Rabi Oscillations in a Josephson Flux Qubit. Phys. Rev. Lett. 91, 097906 (2003).

19 Ya. S. Greenberg, E. Il'ichev, and A. Izmalkov Low-frequency Rabi spectroscopy for a dissipative two-level system. Europhys. Lett. 72, 880 (2005).

20 J. Hauss, A. Fedorov, C. Hutter, A. Shnirman, and G. Schön Single-Qubit Lasing and Cooling at the Rabi Frequency. Phys. Rev. Lett. 100, 037003 (2008).

21 . J. Hauss, A. Fedorov, S. Andre, V. Brosco, C. Hutter, R. Kothari, S. Yeshwanth, A. Shnirman, and G. Schön Dissipation in circuit quantum electrodynamics: lasing and cooling of a low-frequency oscillator. New J. Phys. 10, 095018 (2008)).

${ }^{22}$ M. Grajcar, S. H. W. van der Ploeg, A. Izmalkov, E. Il'ichev, H.-G. Meyer, A. Fedorov, A. Shnirman, and Gerd Schön Sisyphus cooling and amplification by a superconducting qubit. Nature Physics 4, 612 (2008)

${ }^{23}$ P. Macha, S. H. W. van der Ploeg, G. Oelsner, E. Il'ichev, H.G. Meyer, S. Wunsch, and M. Siegel Losses in coplanar waveguide resonators at millikelvin temperatures. Appl. Phys. Lett. 96, 062503 (2010).

${ }^{24}$ A. N. Omelyanchouk, S. N. Shevchenko, Ya. S. Greenberg, O. Astafiev, and E. Il'ichev Quantum behavior of a flux qubit coupled to a resonator. Low Temp. Phys. 36, 893 (2010).

25 G. Oelsner, P. Macha, O. V. Astafiev, E. Il'ichev, M. Grajcar, U. Hübner, B.I. Ivanov, P. Neilinger, and H.-G. Meyer Dressed-State Amplification by a Single Superconducting Qubit. Phys. Rev. Lett. 110, 053602 (2013).

${ }^{26}$ S. N. Shevchenko, G. Oelsner, Ya. S. Greenberg, P. Macha, D. S. Karpov, M. Grajcar, A. N. Omelyanchouk, and E. Il'ichev Amplification and attenuation of a probe signal by doubly dressed states.
Phys. Rev. B89, 184504 (2014).

${ }^{27}$ E. Il'ichev, S. N. Shevchenko, S. H. W. van der Ploeg, M. Grajcar, E. A. Temchenko, A. N. Omelyanchouk, and H.-G. Meyer Multiphoton excitations and inverse population in a system of two flux qubits. Phys. Rev. B81, 012506 (2010).

${ }^{28}$ E. A. Temchenko, S. N. Shevchenko, and A. N. Omelyanchouk Dissipative dynamics of a two-qubit system: Four-level lasing. Phys. Rev. B83, 144507 (2011).

${ }^{29}$ C. Cohen-Tannoudji, J. Dupont-Rock, and G. Grynberg, AtomPhoton Interactions. Basic Principles and Applications, John Wiley, New York (1998), Chap. 6.

${ }^{30}$ M. A. Kmetic, R. A. Thuraisingham, and W. J. Meath Two-level rotating-wave approximations for molecules in a static electric field with an application to rotationally average spactra. Phys. Rev. A33, 1688 (1986).

31 Y. Nakamura, Yu. A. Pashkin, and J. S. Tsai Rabi Oscillations in a Josephson-Junction Charge Two-Level System. Phys. Rev. Lett. 87, 246601 (2001).

${ }^{32}$ W. Krech, D. Born, V. Shnyrkov, Th. Wagner, M. Grajcar, E. Il'ichev, H.-G. Meyer, and Ya. Greenberg Quantum Dynamics of the Interferometer-Type Charge Qubit Under Microwave Irradiation. IEEE Trans Appl. Supercond. 15, 876 (2005).

${ }^{33}$ C. M. Wilson, T. Duty, F. Persson, M. Sandberg, G. Johansson, and P. Delsing Coherence Times of Dressed States of a Superconducting Qubit under Extreme Driving. Phys. Rev. Lett. 98, 257003 (2007).

34 C. M. Wilson, G. Johansson, T. Duty, F. Persson, M. Sandberg Dressed relaxation and dephasing in a strongly driven two-level system. Phys. Rev. B81, 024520 (2010).

35 B. R. Mollow Absorption and Emission Line-Shape Functions for Driven Atoms. Phys. Rev. A5, 1522 (1972).

${ }^{36}$ O. V. Astafiev, K. Inomata, A. O. Niskanen, T. Yamamoto, Yu. A. Pashkin, Y. Nakamura, and J. S. Tsai Single artificial-atom lasing. Nature (London) 449, 588 (2007).

37 O. V. Astafiev, A. A. Abdumalikov, Jr., A. M. Zagoskin, Yu. A. Pashkin, Y. Nakamura, and J. S. Tsai Ultimate On-Chip Quantum Amplifier. Phys. Rev. Lett. 104, 183603 (2010).

${ }^{38}$ K. Koshino, H. Terai, K. Inomata, T. Yamamoto, W. Qiu, Z. Wang, and Y. Nakamura Observation of the Three-State Dressed States in Circuit Quantum Electrodynamics. Phys. Rev. Lett. 110, 263601 (2013).

39 A. Wallraff, D. I. Schuster, A. Blais, J. M. Gambetta, J. Schreier, L. Frunzio, M. H. Devoret, S. M. Girvin, and R. J. Schoelkopf Sideband Transitions and Two-Tone Spectroscopy of a Superconducting Qubit Strongly Coupled to an On-Chip Cavity. Phys. Rev. Lett. 99, 050501 (2007).

40 Y. Shimazu, M. Takahashi, and N. Okamura Observation of Higher-Order Sideband Transitions and First-Order Sideband Rabi Oscillations in a Superconducting Flux Qubit Coupled to a SQUID Plasma Mode. J. Phys. Soc. Jpn. 82, 074710 (2013).

41 A. P. Saiko and R. Fedaruk Multiplication of qubits in a doubly resonant bichromatic field. Pis'ma v ZhETF 91, 750 (2010) [JETP Lett. 91, 681 (2010)].

${ }^{42}$ G. Oelsner, S. H. W. van der Ploeg, P. Macha, U. Hübner, D. Born, S. Anders, E. Il'ichev, H.-G. Meyer, M. Grajcar, S. Wünsch, M. Siegel, A. N. Omelyanchouk, and O. Astafiev Weak continuous monitoring of a flux qubit using coplanar waveguide resonator. Phys. Rev. B81, 172505 (2010). 